\title{
Long Non-Coding RNA MALAT1 Protects Human Osteoblasts from Dexamethasone- Induced Injury via Activation of PPM1E- AMPK Signaling
}

\author{
Jian-bo Fan ${ }^{\mathrm{a}, \mathrm{b}}$ Yingzi Zhang ${ }^{\mathrm{c}}$ Wei Liu ${ }^{\mathrm{a}}$ Xin-hui Zhu ${ }^{\mathrm{a}}$ Da-wei Xu \\ Jian-ning Zhao ${ }^{b}$ Zhi-ming Cui ${ }^{\mathrm{a}}$ \\ aDepartment of Orthopaedics, The Second Affiliated Hospital of Nantong University, Nantong, \\ ${ }^{b}$ Department of Orthopaedics, Jinling Hospital, Nanjing Medical University, Nanjing, 'Department of \\ Orthopedics, the Second Affiliated Hospital of Suzhou University, Suzhou, China
}

\author{
Key Words \\ LnCRNA MALAT1 • Osteoblasts • Dexamethasone • Nrf2
}

\begin{abstract}
Background/Aims: Dexamethasone (Dex) induces injuries to human osteoblasts. In this study, we tested the potential role of the long non-coding RNA metastasis-associated lung adenocarcinoma transcript 1 (Lnc-MALAT1) in this process. Materials: Two established human osteoblastic cell lines (OB-6 and hFOB1.19) and primary human osteoblasts were treated with Dex. Lnc-MALAT1 expression was analyzed by quantitative real-time polymerase chain reaction assay. Cell viability, apoptosis, and death were tested by the MTT assay, histoneDNA assay, and trypan blue staining assay, respectively. AMP-activated protein kinase (AMPK) signaling was evaluated by western blotting and AMPK activity assay. Results: LncMALAT1 expression was downregulated by Dex treatment in the established osteoblastic cell lines (OB-6 and hFOB1.19) and primary human osteoblasts. The level of Lnc-MALAT1 was decreased in the necrotic femoral head tissues of Dex-administered patients. In osteoblastic cells and primary human osteoblasts, forced overexpression of Lnc-MALAT1 using a lentiviral vector (LV-MALAT1) inhibited Dex-induced cell viability reduction, cell death, and apoptosis. Conversely, transfection with Lnc-MALAT1 small interfering RNA aggravated Dex-induced cytotoxicity. Transfection with LV-MALAT1 downregulated Ppm1e (protein phosphatase, $\mathrm{Mg}^{2+1}$ $\mathrm{Mn}^{2+}$-dependent 1e) expression to activate AMPK signaling. Treatment of osteoblasts with AMPK $\alpha 1$ short hairpin RNA or dominant negative mutation (T172A) abolished LV-MALAT1induced protection against Dex-induced cytotoxicity. Furthermore, LV-MALAT1 induced an increase in nicotinamide adenine dinucleotide phosphate activity and activation of Nrf2 signaling. Dex-induced reactive oxygen species production was significantly attenuated
\end{abstract}

J. Fan, W. Liu and Y. Zhang contributed equally to this work.

\begin{tabular}{ll}
\hline Zhi-ming Cui, MD & Department of Orthopaedics, the Second Affiliated Hospital of Nantong University \\
& North Hai-er-xiang Road 6, Nantong, Jiangsu 226001 (China) \\
& Tel. +86-513-85061243, E-Mail zhimingcuiorth@163.com
\end{tabular}


by LV-MALAT1 transfection in osteoblastic cells and primary osteoblasts. Conclusion: LncMALAT1 protects human osteoblasts from Dex-induced injuries, possibly via activation of Ppm1e-AMPK signaling.

(C) 2018 The Author(s)

Published by S. Karger AG, Basel

\section{Introduction}

Dexamethasone (Dex) is prescribed to patients with chronic inflammatory and autoimmune diseases [1]. However, excessive or long-term Dex treatment induces cytotoxic effects in human osteoblasts, serving as a primary cause of osteoporosis and osteonecrosis $[2,3]$. Dex treatment has been shown to exert direct cytotoxic effects on cultured human osteoblasts in vitro [2, 4-7]. Studies focusing on the pathological mechanisms of Dex-induced injuries in osteoblasts have been previously conducted [8-13], and have been the main area of focus of our research group as well $[4,9,11,14]$.

The long non-coding RNA (lncRNA) metastasis-associated lung adenocarcinoma transcript 1 (MALAT1) [15], or Lnc-MALAT1, is an abundant and highly conserved lncRNA [16-18]. It was first discovered as a prognostic marker for lung cancer metastasis [19]. LncMALAT1 is overexpressed in various human cancers [16-18]. It interacts with polycomb-2 to regulate histone modifications and cell proliferation [20]. Furthermore, it interacts with serine/arginine-rich proteins to regulate the subcellular localization of splicing regulatory proteins [21]. Recent studies have proposed the pro-survival function of Lnc-MALAT1 [22]. However, the potential activity of Lnc-MALAT1 in Dex-treated human osteoblasts has not yet been studied.

AMP-activated protein kinase (AMPK) coordinates the signaling of several metabolic pathways $[23,24]$. It is composed of $\alpha-, \beta-$, and $\gamma$-subunits $[23,24]$. Phosphorylation of the $\alpha 1$-subunit at Thr-172 is essential for AMPK activation [23-25]. Activated AMPK is shown to promote cell survival under stress conditions [26]. AMPK can attenuate oxidative stress via activation of nicotinamide adenine dinucleotide phosphate (NADPH) [27] and nuclear factor erythroid 2-related factor 2 (Nrf2) signaling [28-30]. Recent studies have implied that AMPK activation can efficiently protect human osteoblasts from Dex-induced injuries [4, 5, 30, 31].

One strategy to induce AMPK activation is to inhibit the expression of its phosphatases [32], which were largely unknown until recently [33]. Protein phosphatase $\mathrm{Mg}^{2+/} \mathrm{Mn}^{2+}$ dependent (Ppm) 1e (Ppm1e) is an important AMPKo phosphatase [33]. It belongs to the Ppm family $[34,35]$. Our previous study demonstrated that microRNA-135b downregulated Ppm1e expression to activate AMPK signaling, which in turn protected osteoblastic cells from Dex-induced injuries [4]. In the present study, our results suggest that Lnc-MALAT1 protects human osteoblasts from Dex-induced injury via activation of Ppm1e-AMPK signaling.

\section{Materials and Methods}

\section{Chemicals and reagents}

Dexamethasone, puromycin, neomycin, and JC-1 fluorescent dye were purchased from Sigma-Aldrich (St. Louis, MO). Antibodies were purchased from Cell Signaling Technology (Beverly, MA) and Santa Cruz Biotechnology (Santa Cruz, CA). Fetal bovine serum, Dulbecco's modified Eagle's medium, antibiotics, and other cell culture reagents were obtained from Gibco-BRL (Grand Island, NY). The cell lysis buffer was purchased from Sigma-Aldrich (Wuxi, China). TRIzol reagent for RNA assays was obtained from Invitrogen (Shanghai, China). mRNA primers were synthesized by GenePharma Co. (Shanghai, China). The $2^{\prime}, 7^{\prime}$-dichlorofluorescein diacetate (DCF-DA) fluorescent dye for reactive oxygen species (ROS) assay was provided by Roche Diagnostics (Mannheim, Germany). 


\section{Cellular Physiology Cell Physiol Biochem 2018;51:31-45

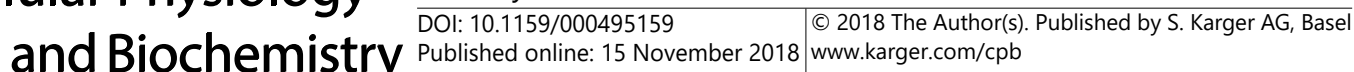

Fan et al.: LncRNA MALAT1 Protects Osteoblasts from Dexamethasone

Cell culture

Established OB-6 [2] and hFOB1.19 [36] human osteoblastic cells were purchased from the Cell Bank of the Shanghai Institute of Biological Sciences (Shanghai, China). Cells were cultured as described previously [2,36]. The primary human osteoblasts were provided by Dr. Ji [6] and cultured under previously described conditions [6,37]. Primary osteoblasts at passage 3-10 were utilized for in vitro experiments. All protocols involving the use of human cells were approved by the Ethics Committee of Nantong University and Declaration of Helsinki.

\section{Quantitative real-time polymerase chain reaction assay}

As described previously [4,38], TRIzol reagent was used to extract total cellular RNA, which was then reverse-transcribed. cDNA was then amplified by quantitative real-time polymerase chain reaction (qPCR). SYBR Green PCR kit (Applied Biosystems, Shanghai, China) was utilized to detect the expression of the listed mRNAs. Melting curve analysis was applied to calculate the product melting temperature. Quantification of mRNA expression was performed using the ${ }^{\Delta} \mathrm{C}$ t method, with glyceraldehyde 3-phosphate dehydrogenase $(G A P D H)$ as the internal control. The primer sequences used for theNrf2 genes, including heme oxygenase-1 (HO1), NAD(P)H quinone oxidoreductase 1 (NQO1), and $\gamma$-glutamyl cysteine ligase catalytic subunit (GCLC) were described previously [39]. Ppm1e mRNA primer sequences were described early [4]. LncMALAT1 primer sequences were as follows: forward: 5' -AAAGCAAGGTCTCCCCACAAG-3' , reverse: 5' -GGTCTGTGCTAGATCAAAAGGCA-3' , as described previously [40]. Lnc-MALAT1 level was normalized to that of $U 6$ mRNA.

\section{Forced Lnc-MALAT1 expression}

The Lnc-MALAT1 sequence was sub-cloned into the lenti-vector-plasmid pCDH-CMV-MCS-EF1-coGFPpuro to generate pCDH-CMV-MALAT1-EF1-coGFP-puro, which was transfected into 293T cells with the lentiviral packaging vectors, psPAX2 and pMD2.G, to produce the lentivirus LV-MALAT1. Human osteoblastic cells or primary osteoblasts were seeded onto 6-well plates at a density of $1 \times 10^{5}$ cells per well. Cells were infected with control virus or LV-MALAT1 for $48 \mathrm{~h}$, followed by culture in puromycin $(1 \mu \mathrm{g} / \mathrm{mL})$-containing medium for another 2-3 weeks. The expression of Lnc-MALAT1 in stable cells was determined by qPCR assay.

\section{Lnc-MALAT1 small interfering RNA}

Small interfering RNAs (siRNAs) targeting Lnc-MALAT1,including siMALAT1-1(5' GAAUUCCGGUGAUGCGAGU-3' ) and siMALAT1-2 (5' -GGCAAUAGAGGCCCUCUAA-3'), were obtained from GenePharma Co. Human osteoblastic cells or primary osteoblasts were seeded onto 6-well tissue culture plates at a density of $1 \times 10^{5}$ cells per well. Cells were transfected with $500 \mathrm{nM}$ of Lnc-MALAT1 siRNA or scramble control siRNA (siR-C) by Lipofectamine 2000 (Invitrogen) for 48 h.Lnc-MALAT1 knockdown efficiency was determined by qPCR assay.

\section{Western blotting}

As described in our previous studies $[9,11]$, total cell lysates were separated bydenaturing on $10 \%$ polyacrylamide gels [41], and then transferred to polyvinylidene difluoride membranes (Millipore, Shanghai, China). The membranes were blocked with $10 \%$ non-fat dry milk, followed by incubation with specific primary and secondary antibodies. The antigen-antibody binding was detected using enhanced chemiluminescence (ECL) reagents (Pierce, Shanghai, China). ImageJ software was utilized for quantification of the total gray area of each protein band.

\section{Cell death assay}

Human osteoblastic cells or primary osteoblasts were seeded onto 12 -well plates at a density of $5 \times 10^{4}$ cells per well. Following treatment with Dex, cells were trypsinized. Cells were stained with trypan blue, and cell counting was performed using a cytometer. The cell death percentage was then calculated using the following formula: Cell death percentage (\%) = (number of trypan blue-stained cells)/(number of total cells) [11]. 


\section{Cellular Physiology Cell Physiol Biochem 2018;51:31-45 and Biochemistry \begin{tabular}{l|l} 
DOI: 10.1159/000495159 & (c) 2018 The Author(s). Published by S. Karger AG, Basel \\
wublisheg
\end{tabular}

Fan et al.: LncRNA MALAT1 Protects Osteoblasts from Dexamethasone

Cell viability assay

Human osteoblastic cells or primary osteoblasts were seeded onto 96-well plates at a density of $3 \times 10^{3}$ cells per well. Following the treatment with Dex, cell viability was assessed by the routine 3-[4, 5-dimethylthylthiazol-2-yl]-2, 5 diphenyltetrazolium bromide (MTT) dye assay [9, 11]. MTT optical densities (ODs) at $550 \mathrm{~nm}$ were recorded.

\section{Detection of apoptosis by enzyme-linked immunosorbent assay}

Human osteoblastic cells or primary osteoblasts were seeded onto 96 -well plates $\left(3 \times 10^{3}\right.$ cells per well). After the applied treatment, a histone-DNA enzyme-linked immunosorbent assay (ELISA) kit (Roche, Palo Alto, CA) was used to quantify cell apoptosis [42]. Histone-DNA ELISA OD at $450 \mathrm{~nm}$ was recorded.

\section{AMPK $\alpha 1$ knockdown}

AMPK $\alpha 1$ short hairpin RNA (shRNA) was used as described in our previous study [42]. Human osteoblastic cells or primary osteoblasts were seeded onto 6-well plates. The lentiviral AMPK $\alpha 1$ shRNA construct ( $10 \mu \mathrm{L}$ virus $/ \mathrm{mL}$ ) was added directly to the cells for $24 \mathrm{~h}$. Cells were cultured in puromycin (1 $\mu \mathrm{g} / \mathrm{mL}$ )-containing medium for 2-3 weeks. Knockdown of AMPK $\alpha 1$ in stable cells was verified by western blotting.

\section{AMPK dominant negative mutation}

Cells were seeded onto 6 -well plates at a density of $1 \times 10^{5}$ cells per well. The dominant negative mutant of AMPK $\alpha 1$ (dn-AMPK $\alpha 1$; T172A; Flag-tagged; provided by Dr. Lu [43]) was transfected into cultured cells using Lipofectamine 2000 [43]. Stable cells were selected by neomycin ( $2 \mu \mathrm{g} / \mathrm{mL}$; Sigma-Aldrich). The expression of dn-AMPK $\alpha 1$ was confirmed by western blotting.

\section{AMPK activity assay}

Following treatment, $500 \mu \mathrm{g}$ of total cell lysate proteins were incubated with anti-AMPK $\alpha 1$ antibody (Cell Signaling Technology). As described previously, AMPK activity was measured in kinase assay buffer usingthe AMP- $\left[\gamma^{-32} \mathrm{P}\right]$ ATP mixture along with the SAMS (HMRSAMSGLHLVKRR) peptide [44]. To stop the reaction, phosphocellulose paper was added. AMPK radioactivity was measured with a scintillation counter.

\section{NADPH activity assay}

NADPH activity was testedby a previously described method [45]. Following treatment, intracellular $\mathrm{NADPH}$ and $\mathrm{NADP}^{+}$levels were examined [46]. Briefly, two million cells were plated onto $10 \mathrm{~cm}$ dishes and lysed in $300 \mu \mathrm{L}$ of extraction buffer after the applied treatment [27]. For NADPH extraction, $100 \mu \mathrm{L}$ of the supernatant was incubated at $60^{\circ} \mathrm{C}$ for $30 \mathrm{~min}$. Next, $100 \mu \mathrm{L}$ of NADP-cycling buffer [27] with $1.0 \mathrm{U}$ glucose-6-phosphate dehydrogenase (G6PD; Sigma-Aldrich) was added. After 1 min of incubation at $30{ }^{\circ} \mathrm{C}, 20 \mu \mathrm{L}$ of $10 \mathrm{mM}$ glucose 6-phosphate (G6P; Sigma-Aldrich) was added to the mixture, and the change in absorbance at $570 \mathrm{~nm}$ was measured. The concentration of $\mathrm{NADP}^{+}$was calculated by subtracting [NADPH] from [total NADP] [27]. The relative NADPH activity was calculated by determining the $\mathrm{NADPH} / \mathrm{NADP}^{+}$ratio.

\section{ROS measurement}

Cells were seeded onto 12 -well plates at a density of $5 \times 10^{4}$ cells per well. Following treatment, ROS production was measured bythe DCF-DA fluorescent dye assay. Cells were incubated with $5 \mu \mathrm{M}$ of DCF-DA for $30 \mathrm{~min}$. Fluorescence intensity was measured using a flow cytometer (BD Biosciences, Shanghai, China).

\section{Mitochondrial depolarization assay}

After the applied treatment, mitochondrial depolarization (" $\Delta \Psi$ ") was tested using the mito-dye JC1 , which aggregates to form green monomers after mitochondrial depolarization [47]. Cells were initially seeded onto 24-well tissue culture plates. The protocol for mitochondrial depolarization assayusing JC-1 has been discussed previously [48]. JC-1 fluorescence ODs were recorded at $550 \mathrm{~nm}$. 


\section{Cellular Physiology Cell Physiol Biochem 2018:51:31-45 and Biochemistry \begin{tabular}{l|l} 
DOI: 10.1159/000495159 & (c) 2018 The Author(s). Published by S. Karger AG, Basel \\
wublisarger.com/cpb
\end{tabular}

\section{Human tissue collection and analysis}

As described previously [4], surgery-isolated necrotic femoral head tissues and thesurrounding normal femoral head tissues were collected from patients who were taking Dex and undergoing femoral head resection. Fresh tissues were dissolved in tissue lysis buffer. The protocols were approved by the Ethics Committee of Nantong University. Written informed consent was obtained from each patient. A total of nine patients were included. All clinical investigations were conducted according to the criteria set by the Declaration of Helsinki.

\section{Statistical analysis}

All quantitative data are presented are the mean \pm standard deviation (SD) from at least three samples per data point. Statistical differences between groups were determined by one-way analysis of variance followed by multiple comparisons performed using Bonferroni post-hoc test. All analyses were performed with SPSS version 18.0 statistical software (SPSS Co., Chicago, IL). Values of $p<0.05$ were considered statistically significant.

\section{Results}

Lnc-MALAT1 expression is downregulated in Dex-treated human osteoblasts and in the necrotic femoral head tissues of Dex-administered patients

To test whether Dex can affect Lnc-MALAT1 expression, two established human osteoblasticcell lines, OB-6 and hFOB1.19, were treated with Dex $(1 \mu \mathrm{M})[4,5]$. After $12 \mathrm{~h}$ of incubation, Lnc-MALAT1 expression level was significantly decreased in OB-6 (Fig. 1A) and hF0B1.19 cells (Fig. 1B), as well as in the primary human osteoblasts (Fig. 1C). Similarly, Lnc-MALAT1 level was also reduced in the necrotic femoral head tissues of Dex-administered patients [4]. However, its level in the paired surrounding normal femoral head tissues was relatively high (Fig. 1D).

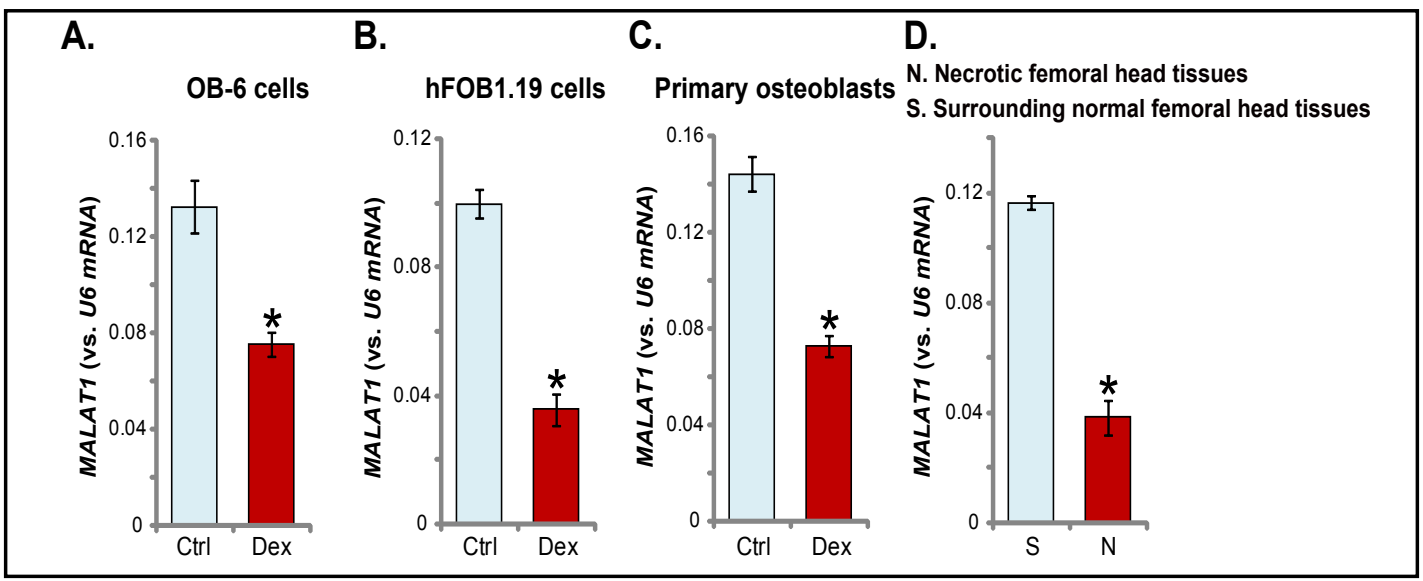

Fig. 1. Lnc-MALAT1 expression is downregulated in Dex-treated human osteoblasts and in the necrotic femoral head tissues of Dex-administered patients. Human osteoblastic OB-6 (A) and hFOB1.19 cells (B) or primary human osteoblasts $(\mathrm{C})$ were treated with or without Dex $(1 \mu \mathrm{M})$ for $12 \mathrm{~h}$. Lnc-MALAT1 expression (vs. U6 mRNA) was analyzed (A-C). qPCR analysis of the relative Lnc-MALAT1 expression (vs. U6 mRNA) in the surgery-isolated femoral head tissues (both normal and necrotic) from a total of nine Dex-administered patients (D). "Ctrl" represents untreated control group. All quantitative data are presented as the mean \pm SD (same for all figures). ${ }^{*} \mathrm{p}<0.05$ vs. Ctrl cells (A-C; $n=5$ ). ${ }^{*} \mathrm{p}<0.05$ vs. "S" tissues (surrounding normal femoral head tissues) ( $D ; n=9)$. Experiments in this figure were repeated three times, and similar results were obtained. 
A.

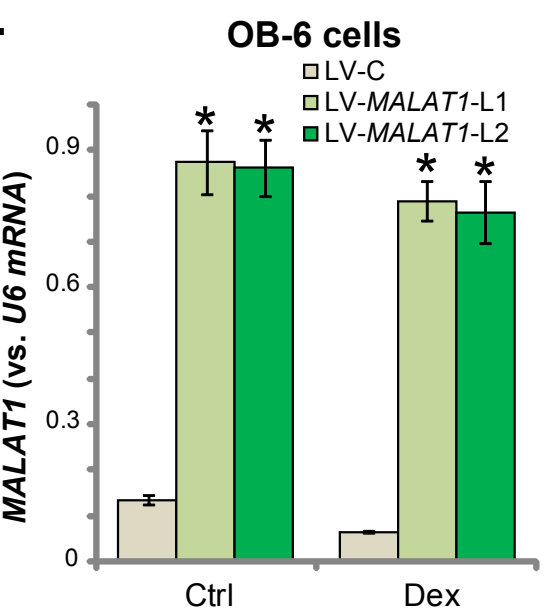

C.

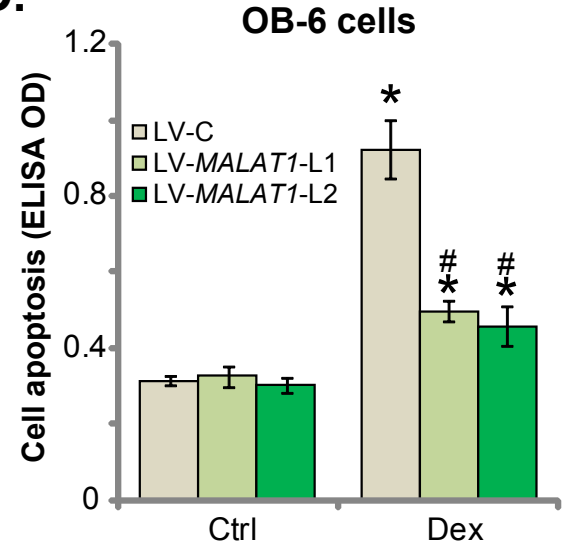

E.

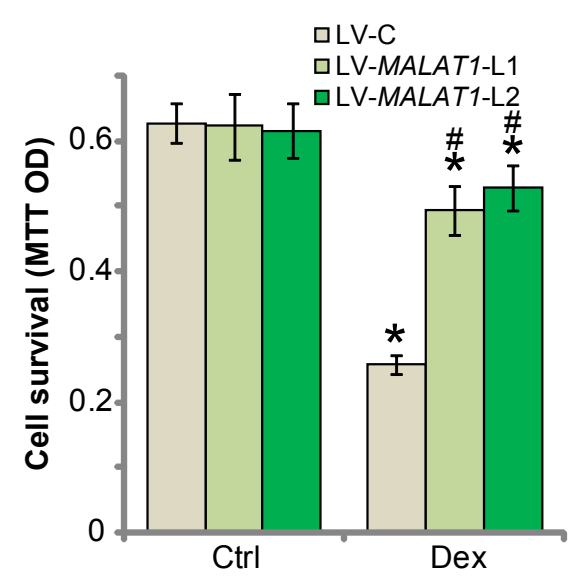

B. OB-6 cells

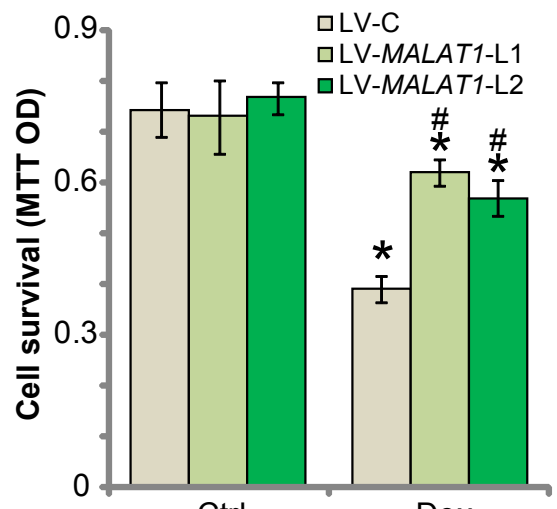

D.

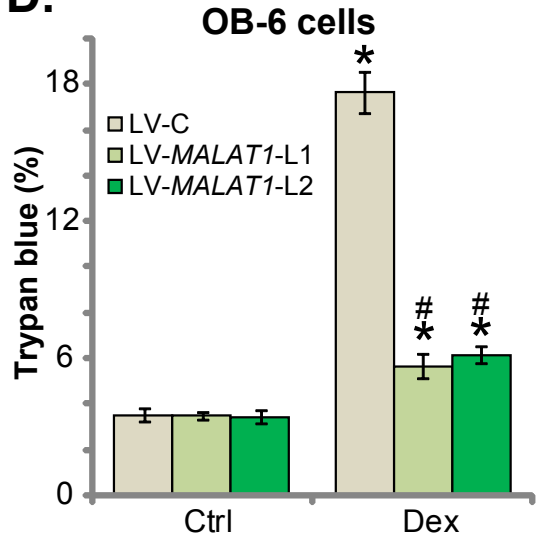

F.

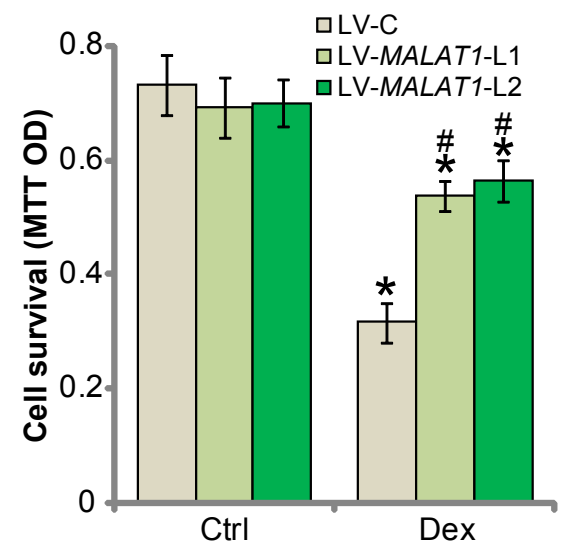

Fig. 2. Forced overexpression of Lnc-MALAT1 protects human osteoblasts from Dex-induced injuries. Stable osteoblastic OB-6 (A-D) and hFOB1.19 cells (E) or primary human osteoblasts (F) transfected with Lnc-MALAT1-expressing lentivirus (two stable lines:LV-MALAT1-L1/LV-MALAT1-L2) or non-sense control lentivirus (LV-C), were treated with or without Dex $(1 \mu \mathrm{M})$ for $24 \mathrm{~h}$. Lnc-MALAT1 expression (vs. U6 mRNA) was analyzed (A).Cell viability (MTT assay; B, E, and F), cell apoptosis (histone-DNA ELISA assay; C), and cell death (trypan blue staining assay; D) were evaluated. For each assay, $n=5$. "Ctrl" represents untreated control group. ${ }^{*} \mathrm{p}<0.05$ vs. Ctrl cells. ${ }^{*} \mathrm{p}<0.05$ vs.LV-C cells with Dex treatment. Experiments in this figure were repeated four times, and similar results were obtained. 


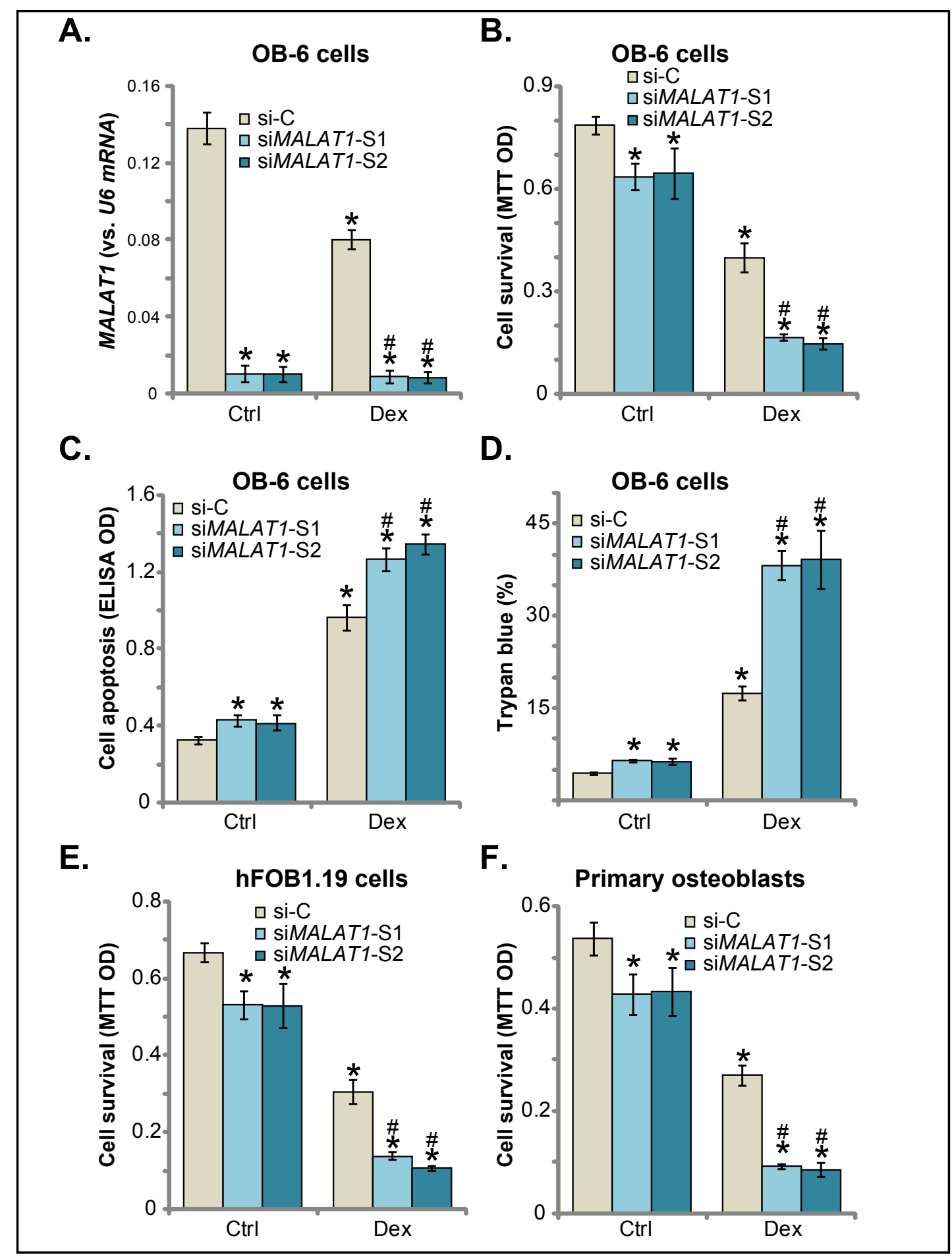

Fig. 3. Lnc-MALAT1 silencing aggravates Dex-induced cytotoxicity in human osteoblasts. Human osteoblastic OB-6 (A-D) and hFOB1.19 cells (E) or primary human osteoblasts (F) were transfected with Lnc-MALAT1 siRNA (siMALAT1-S1/S2; $500 \mathrm{nM}$, for $48 \mathrm{~h}$ ) or scramble control siRNA (si-C; $500 \mathrm{nM}$, for $48 \mathrm{~h}$ ). Cells were then treated with Dex $(1 \mu \mathrm{M})$ for $24 \mathrm{~h}$. Lnc-MALAT1 expression (vs. U6 mRNA) was analyzed (A). Cell viability (MTT assay; B, E, and F), cell apoptosis (histone-DNA ELISA assay; C), and cell death (trypan blue staining assay; D) were evaluated. For each assay, $n=5$. "Ctrl” represents untreated control group. *p<0.05 vs. Ctrl cells. ${ }^{\#}$ p 0.05 vs. Dex-treated si-C cells. Experiments in this figure were repeated four times, and similar results were obtained. 


\section{Cellular Physiology Cell Physiol Biochem 2018;51:31-45 and Biochemistry Publisned onIne: 15 November $2018 \begin{aligned} & \text { (c) } 2018 \text { The Author(s). Published by S. Karger AG, Basel } \\ & \text { www.karger.com/cpb }\end{aligned}$ \\ Fan et al.: LncRNA MALAT1 Protects Osteoblasts from Dexamethasone}

Forced overexpression of Lnc-MALAT1 protects human osteoblasts from Dex-induced injuries

Lnc-MALAT1 expression was downregulated following Dex treatment in human osteoblasts, indicating its potential activity in Dex-induced cytotoxicity. Next, a lentiviral Lnc-MALAT1 expression construct (LV-MALAT1) was transfected into OB-6 cells. Following puromycin selection, two stable OB-6 cell lines were established: LV-MALAT1-L1 and LVMALAT1-L2. By performing qPCR assay, we showed that Lnc-MALAT1 expression level was significantly increased in the stable cells transfected with LV-MALAT1, even after Dex treatment (Fig. 2A). Additionally, Dex-induced viability reduction (MTT OD) was attenuated following LV-MALAT1 transfection in OB-6 cells (Fig. 2B). Furthermore, results from HistoneDNA ELISA apoptosis assay and trypan blue staining assay showed that Dex-induced cell apoptosis and death were significantly inhibited in stable OB-6 cells transfected with LVMALAT1 (Fig. 2C and D). Treatment with LV-MALAT1 alone failed to affect OB-6 cell survival, death, or apoptosis (Fig. 2B-D).

In hFOB1.19 osteoblastic cells, forced overexpression of Lnc-MALAT1 attenuated Dex-induced viability reduction (Fig. 2E). In primary human osteoblasts, cells transfected with LV-MALAT1 were protected from Dex-induced injury (Fig. 2F). Together, these results indicate that forced overexpression of Lnc-MALAT1 protects human osteoblasts from Dexinduced injury.

Lnc-MALAT1 silencing aggravates Dex-induced cytotoxicity in human osteoblasts

Next, we hypothesized that Lnc-MALAT1 silencing enhances Dex-induced cytotoxicity. To test this hypothesis, Lnc-MALAT1 siRNAs (siMALAT1-S1/S2) were transfected into OB-6 cells. As shown in Fig. 3A, each of the applied siRNA induced over $90 \%$ reduction of LncMALAT1 expression in 0B-6 cells. Lnc-MALAT1 siRNA induced minor but significant viability reduction (Fig. 3B), apoptosis (Fig. 3C), and death (Fig. 3D) in OB-6 cells. Additionally, Lnc-MALAT1 silencing aggravated Dex-induced cytotoxicity, thereby enhancing viability reduction (Fig. 3B), cell apoptosis (Fig. 3C), and death (Fig. 3D). In hFOB1.19 osteoblastic cells and primary human osteoblasts, similar results were obtained. Lnc-MALAT1 siRNA transfection significantly enhanced Dex-induced viability reduction (Fig. 3E and F). LncMALAT1 silencing alone only slightly inhibited the viability of hFOB1.19 cells and primary osteoblasts (Fig. 3E and F). These results further support the role of Lnc-MALAT1 in Dexinduced osteoblast injury.

AMPK activation mediates LV-MALAT1-induced protection against Dex-induced cytotoxicity

Our previous study showed that activation of Ppm1e-AMPK signaling could protect human osteoblasts from Dex-induced injuries [4]. The potential effect of Lnc-MALAT1 on this signaling was tested. By performing qPCR assay, we showed that transfection with LVMALAT1 decreasedPpm1e mRNA expression in OB-6 cells (Fig. 4A). Ppm1e protein expression was also downregulated, causing increased phosphorylation of AMPK $\alpha 1$ (at Thr-172) and its major downstream protein, acetyl-coA carboxylase (ACC; at Ser-79) (Fig. 4B). Further studies showed that AMPK activity was also increased in LV-MALAT1-expressing OB-6 cells (Fig. 4C). These results suggestthat forced overexpression of Lnc-MALAT1 activates AMPK signaling in OB-6 cells.

Next, genetic strategies were employed to inhibit AMPK activation. As described previously [4], OB-6 cells were treated with AMPK $\alpha 1$ shRNA lentivirus (sh-AMPK $\alpha 1$ ) to stably knockdown AMPK $\alpha 1$ (Fig. 4D). Additionally, a dominant negative mutant of AMPK $\alpha 1$ (dn-AMPK $\alpha 1$; T172A) was transfected into OB-6 cells (Fig. 4D). As demonstrated in Fig. 4D, transfection with sh-AMPK $\alpha 1$ or dn-AMPK $\alpha 1$ almost completely blocked LV-MALAT1induced AMPK activation (AMPK $\alpha 1-A C C$ phosphorylation) in OB-6 cells. Moreover, LVMALAT1 treatment was ineffective against Dex-induced injury in 0B-6 cells transfected with sh-AMPK $\alpha 1$ or dn-AMPK $\alpha 1$ (Fig. 4E and F). Therefore, when AMPK $\alpha 1$ was silenced or mutated, LV-MALAT1 failed to inhibit Dex-induced OB-6 cell injuries. These results suggest 


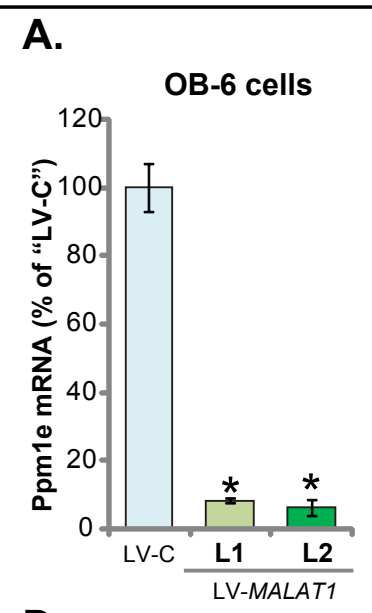

D.

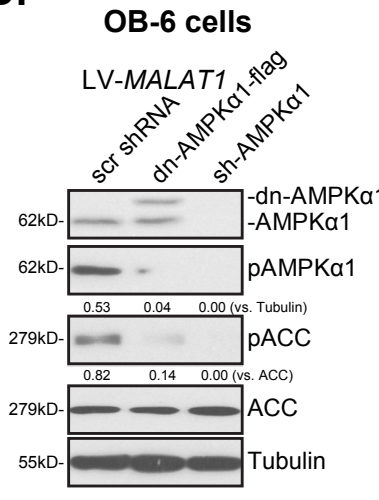

G.

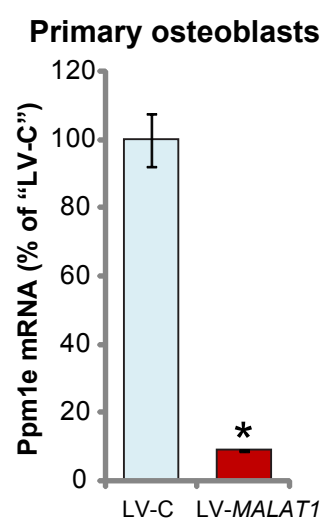

B.

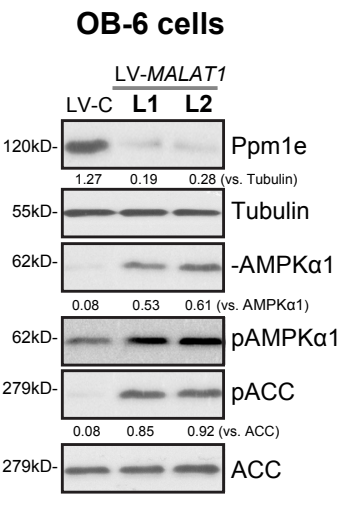

E.

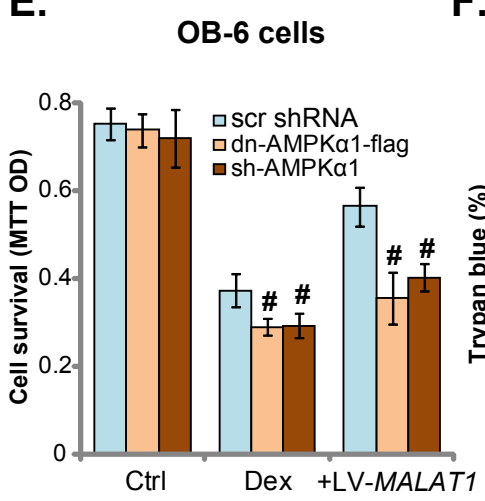

C.

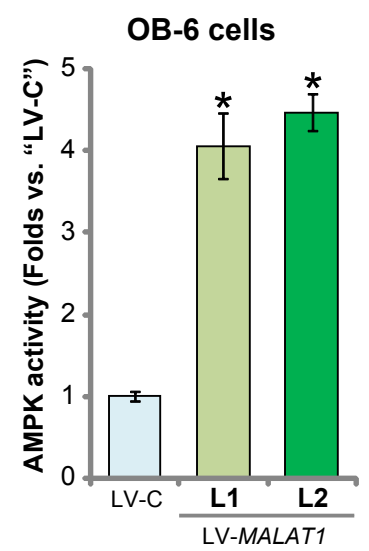

F.

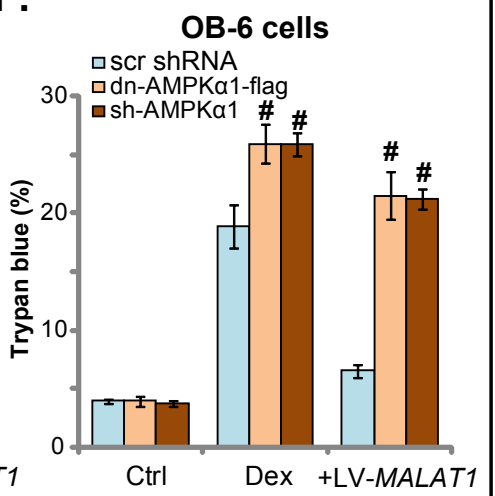

H.

Primary osteoblasts

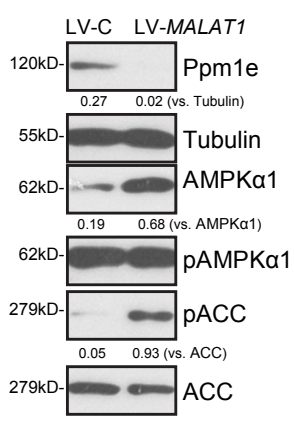

I.

\section{Primary osteoblasts}

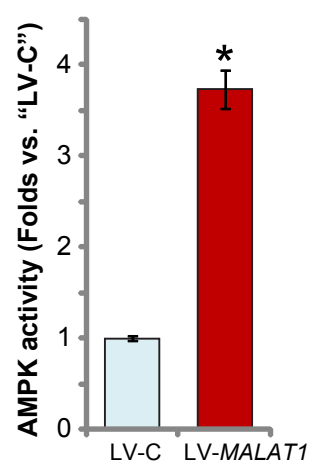

Fig. 4. AMPK activation mediates LV-MALAT1-induced osteoblast cytoprotection against Dex-induced injuries. The expression of Ppm1e mRNA ( $A$ and $G$ ) and listed proteins ( $B$ and $H$ ), and relative AMPK activity (C and I) were assessed in stable OB-6 cells (A-C) or primary human osteoblasts (G-I) transfected with Lnc-MALAT1-expressing lentivirus (LV-MALAT1) or non-sense control lentivirus (LV-C). Stable OB-6 cells transfected with AMPK $\alpha 1$ shRNA (sh-AMPK $\alpha 1$ ), dominant negative AMPK $\alpha 1$ (dn-AMPK $\alpha 1$-flag, T172A), or scramble control shRNA (scr shRNA), were infected with LV-MALAT1 for $48 \mathrm{~h}$, and the expression of listed proteins was analyzed (D).Cells were further treated with Dex $(1 \mu \mathrm{M})$ for $24 \mathrm{~h}$, and cell viability (MTT assay; E) and cell death (trypan blue staining assay; F) were evaluated. Expression of the listed proteins was quantified (B, D, and H). For each assay, $\mathrm{n}=5$. "Ctrl" represents untreated control group. ${ }^{*} \mathrm{p}<0.05$ vs. LV-C cells (A, C, G, and I). " $p<0.05$ vs. scr shRNA cells ( $E$ and F). Experiments in this figure were repeated three times, and similar results were obtained. 


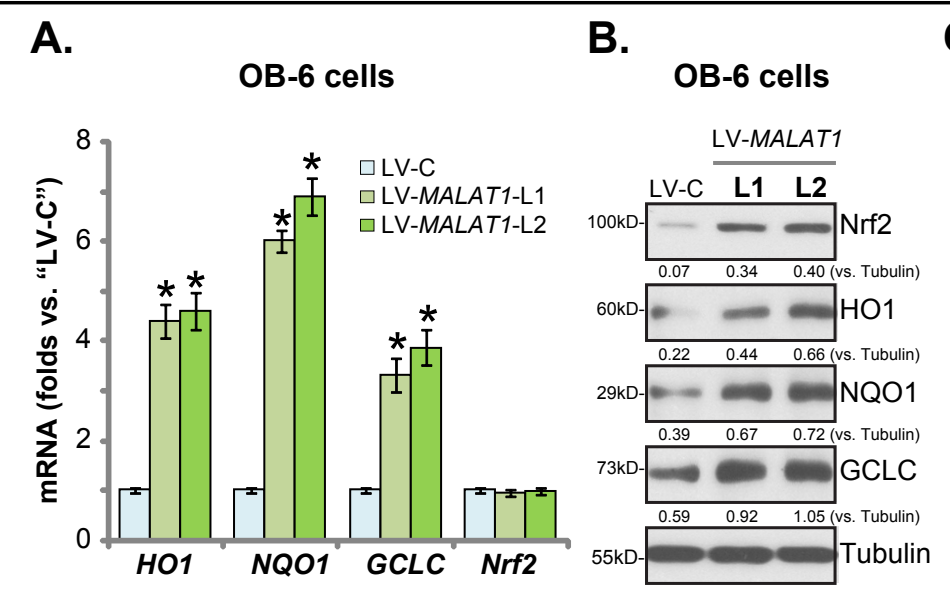

C.

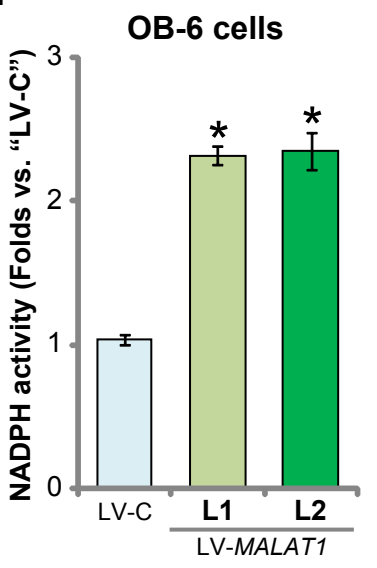

D.

E.
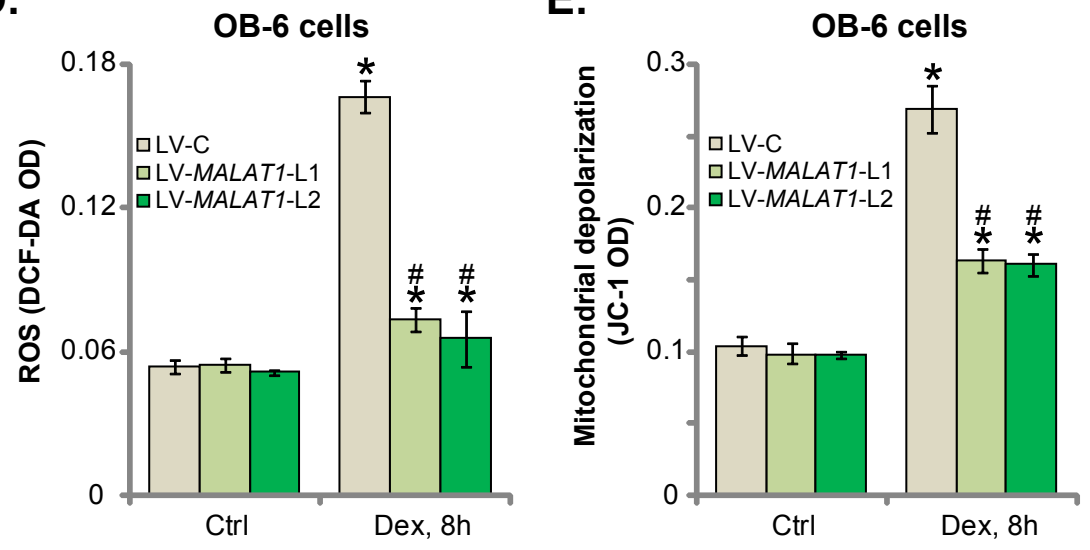

$\mathbf{F}$.

G.

Primary osteoblasts

Primary osteoblasts
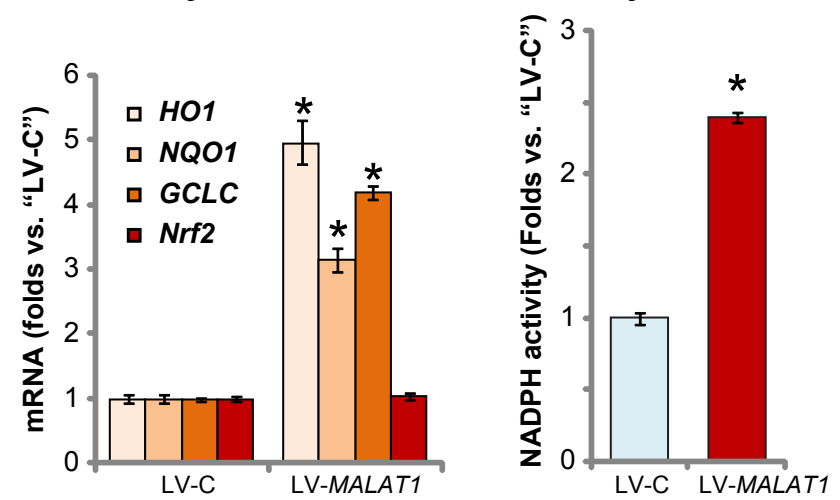

Fig. 5. Lnc-MALAT1 inhibits Dex-induced oxidative stress in human osteoblasts. The expression of listed mRNAs (A and F) and proteins (B), and relative NADPH activity (C and G) were assessed in stable osteoblastic OB-6 cells (A-C) or primary human osteoblasts (F and G) transfected with Lnc-MALAT1expressing lentivirus (LV-MALAT1) or non-sense control lentivirus (LV-C). Cells were further treated with Dex $(1 \mu \mathrm{M})$ for $8 \mathrm{~h}$, and relative ROS production and mitochondrial depolarization were tested by DCF-DA fluorescence dye assay (D) and JC-1 fluorescence dye assay (E), respectively. Expression of listed proteins was quantified (B). "Ctrl" represents untreated control group. *p $<0.05$ vs. LV-C cells (A, C, F, and G). ${ }^{*}$ p $<0.05$ vs. Ctrl cells (D and E). ${ }^{*} \mathrm{p}<0.05$ vs. Dex-treated LV-C cells (D and E). Experiments in this figure were repeated three times, and similar results were obtained. 
that AMPK activation is required for LV-MALAT1-induced osteoblast cytoprotection against Dex-induced injuries. In primary human osteoblasts, transfection with LV-MALAT1 similarly induced Ppm1e downregulation (Fig. 4G and H), AMPKo1-ACC phosphorylation (Fig. 4H), and AMPK activation (Fig. 4I).

\section{Lnc-MALAT1 inhibits Dex-induced oxidative stress in human osteoblasts}

Dex induces ROS production to mediate osteoblast injuries [4, 30, 31]. Studies have shown that AMPK activation can inhibit Dex-induced ROS production [4, 30, 31, 49, 50]. Activated AMPK can increase NADPH content and activity to attenuate oxidative stress $[49,51]$. AMPK can also induce activation of Nrf2 signaling [28-30], which is a key cellular defense mechanism against oxidative stress [52-54]. In OB-6 cells, transfection with LVMALAT1 significantly enhanced the mRNA and protein expression of several key Nrf2dependent enzymes, including HO1, NOQ1, and GCLC (Fig. 5A and B). Nrf2 mRNA level was not significantly altered by LV-MALAT1 transfection in OB-6 cells (Fig. 5A). Nrf2 protein level was, however, significantly increased (Fig. 5B). These results indicate that LV-MALAT1 activates Nrf2 signaling in OB-6 cells.

Further studies showed that in LV-MALAT1-expressing OB-6 cells, NADPH activity was enhanced (Fig. 5C), while Dex-induced increase in ROS production or DCF-DA intensity was attenuated (Fig. 5D). Additionally, Dex-induced mitochondrial depolarization (JC-1 OD increase) was also attenuated (Fig. 5E). These results suggest that LV-MALAT1 activates Nrf2 and NADPH signaling, thereby inhibiting Dex-induced oxidative stress. In primary human osteoblasts, transfection with LV-MALAT1 similarly enhanced the mRNA expression of Nrf2dependent genes (Fig. 5F) as well as NADPH activity (Fig. 5G).

\section{Discussion}

Prolonged and/or excessive Dex usage can cause secondary osteoporosis [55, 56] or osteonecrosis [57]. Reduced number of osteoblasts, decreased osteoblastogenesis, and increased osteoblast apoptosis were detected in the bones of Dex-administered patients [55, 56]. In the present study, in both osteoblastic cells (OB-6 and hFOB1.19 lines) and primary human osteoblasts, overexpression of Lnc-MALAT1 by LV-MALAT1 inhibited Dex-induced viability reduction, cell death, and apoptosis. Conversely, transfection with Lnc-MALAT1 siRNA aggravated Dex-induced injuries. Lnc-MALAT1 overexpression downregulated Ppm1e expression to activate AMPK signaling. Conversely, transfection with AMPK 1 shRNA or its dominant negative mutation almost abolished Lnc-MALAT1-mediated osteoblast cytoprotection. Thus, Lnc-MALAT1 protects human osteoblasts from Dex-induced injury possibly via activation of Ppm1e-AMPK signaling.

AMPK activation can inhibit ROS production and oxidative injury [27, 51]. AMPK activation is essential for NADPH homeostasis [27, 51]. Activated AMPK phosphorylates ACC to prevent NADPH consumption [27]. Furthermore, fatty-acid oxidation by AMPK promotes NADPH synthesis [27]. Recent studies have proposed another mechanism to explain AMPK-induced antioxidant capacity, which involves activation of Nrf2 signaling. Nrf2 binds tokelch-like ECH-associated protein 1 (Keap1), an adaptor protein, which is responsible for cullin 3-dependent Nrf2 ubiquitination and proteasomal degradation [53, $58,59]$. Activated Nrf2 disassociates with Keap1, which leads to Nrf2 protein accumulation, nuclear translocation, and activation [53, 58, 59]. AMPK is shown to directly phosphorylate Nrf2 (at Ser-550) to increase Nrf2 nuclear translocation and activation [29]. Zimmermann et al. previously reported a signaling crosstalk between AMPK and Nrf2 [28]. Compound 991 and PF-06409577, two well-known AMPK activators, have also been shown to activate Nrf2 signaling [30, 45]. 
Fan et al.: LncRNA MALAT1 Protects Osteoblasts from Dexamethasone

In this study, we showed that transfection with LV-MALAT1 increased NADPH activity and the expression of Nrf2-dependent genes, and attenuated Dex-induced ROS production in human osteoblastic cells and primary osteoblasts. Therefore, Lnc-MALAT1 could provoke AMPK-dependent antioxidant mechanisms to protect human osteoblasts from Dex-induced injury [22].

\section{Conclusion}

Our results suggest that Lnc-MALAT1 protects human osteoblasts from Dex-induced injuries possibly via activation of Ppm1e-AMPK signaling.

\section{Acknowledgements}

This work was generously supported by grants from the National Natural Science Foundation of China (81501866), Jiangsu Provincial Young Medical Talent Foundation (QNRC2016411), the Science and Technology Program of Nantong (MS22015051), and Nantong 226 High-level Talents Project and Jiangsu 333 Talent Peak Program (To J. F.).

\section{Disclosure Statement}

None of the authors has any competing interests to declare.

\section{References}

1 Schacke H, Docke WD, Asadullah K: Mechanisms involved in the side effects of glucocorticoids. Pharmacol Ther 2002;96:23-43.

-2 Ding H, Wang T, Xu D, Cha B, Liu J, Li Y: Dexamethasone-induced apoptosis of osteocytic and osteoblastic cells is mediated by TAK1 activation. Biochem Biophys Res Commun 2015;460:157-163.

-3 Yun SI, Yoon HY, Jeong SY, Chung YS: Glucocorticoid induces apoptosis of osteoblast cells through the activation of glycogen synthase kinase 3beta. J Bone Miner Metab 2009;27:140-148.

4 Fan JB, Ruan JW, Liu W, Zhu LQ Zhu XH, Yi H, Cui SY, Zhao JN, Cui ZM: miR-135b expression downregulates Ppm1e to activate AMPK signaling and protect osteoblastic cells from dexamethasone. Oncotarget 2016;7:70613-70622.

5 Guo S, Chen C, Ji F, Mao L, Xie Y: PP2A catalytic subunit silence by microRNA-429 activates AMPK and protects osteoblastic cells from dexamethasone. Biochem Biophys Res Commun 2017;487:660-665.

-6 Zhao S, Mao L, Wang SG, Chen FL, Ji F, Fei HD: MicroRNA-200a activates Nrf2 signaling to protect osteoblasts from dexamethasone. Oncotarget 2017;8:104867-104876.

-7 Liu G, Chen FL, Ji F, Fei HD, Xie Y, Wang SG: microRNA-19a protects osteoblasts from dexamethasone via targeting TSC1. Oncotarget 2018;9:2017-2027.

-8 Ji F, Mao L, Liu Y, Cao X, Xie Y, Wang S, Fei H: K6PC-5, a novel sphingosine kinase 1 (SphK1) activator, alleviates dexamethasone-induced damages to osteoblasts through activating SphK1-Akt signaling. Biochem Biophys Res Commun 2015;458:568-575.

-9 Fan JB, Liu W, Zhu XH, Yuan K, Xu DW, Chen JJ, Cui ZM: EGFR-AKT-mTOR activation mediates epiregulininduced pleiotropic functions in cultured osteoblasts. Mol Cell Biochem 2015;398:105-113.

10 Zhen YF, Wang GD, Zhu LQ Tan SP, Zhang FY, Zhou XZ, Wang XD: P53 dependent mitochondrial permeability transition pore opening is required for dexamethasone-induced death of osteoblasts. J Cell Physiol 2014;229:1475-1483.

11 Fan JB, Liu W, Yuan K, Zhu XH, Xu DW, Chen JJ, Cui ZM: EGFR trans-activation mediates pleiotrophininduced activation of Akt and Erk in cultured osteoblasts. Biochem Biophys Res Commun 2014;447:425430. 


\section{Cellular Physiology Cell Physiol Biochem 2018:51:31-45 and Biochemistry \begin{tabular}{l|l} 
DOI: 10.1159/000495159 & (c) 2018 The Author(s). Published by S. Karger AG, Basel
\end{tabular}

Fan et al.: LncRNA MALAT1 Protects Osteoblasts from Dexamethasone

12 Plotkin LI, Weinstein RS, Parfitt AM, Roberson PK, Manolagas SC, Bellido T: Prevention of osteocyte and osteoblast apoptosis by bisphosphonates and calcitonin. J Clin Invest 1999;104:1363-1374.

13 Gohel A, McCarthy MB, Gronowicz G: Estrogen prevents glucocorticoid-induced apoptosis in osteoblasts in vivo and in vitro. Endocrinology 1999;140:5339-5347.

14 Guo S, Xie Y, Fan JB, Ji F, Wang S, Fei H: alpha-Melanocyte stimulating hormone attenuates dexamethasoneinduced osteoblast damages through activating melanocortin receptor 4-SphK1 signaling. Biochem Biophys Res Commun 2016;469:281-287.

15 Guo D, Ma J, Yan L, Li T, Li Z, Han X, Shui S: Down-Regulation of Lncrna MALAT1 Attenuates Neuronal Cell Death Through Suppressing Beclin1-Dependent Autophagy by Regulating Mir-30a in Cerebral Ischemic Stroke. Cell Physiol Biochem 2017;43:182-194.

16 Amodio N, Raimondi L, Juli G, Stamato MA, Caracciolo D, Tagliaferri P, Tassone P: MALAT1: a druggable long non-coding RNA for targeted anti-cancer approaches. J Hematol Oncol 2018;11:63.

17 Toraih EA, Ellawindy A, Fala SY, Al Ageeli E, Gouda NS, Fawzy MS, Hosny S: Oncogenic long noncoding RNA MALAT1 and HCV-related hepatocellular carcinoma. Biomed Pharmacother 2018;102:653-669.

18 Zhang X, Hamblin MH, Yin KJ: The long noncoding RNA Malat1: Its physiological and pathophysiological functions. RNA Biol 2017;14:1705-1714.

19 Ji P, Diederichs S, Wang W, Boing S, Metzger R, Schneider PM, Tidow N, Brandt B, Buerger H, Bulk E, Thomas M, Berdel WE, Serve H, Muller-Tidow C: MALAT-1, a novel noncoding RNA, and thymosin beta4 predict metastasis and survival in early-stage non-small cell lung cancer. Oncogene 2003;22:8031-8041.

-20 Yang L, Lin C, Liu W, Zhang J, Ohgi KA, Grinstein JD, Dorrestein PC, Rosenfeld MG: ncRNA- and Pc2 methylation-dependent gene relocation between nuclear structures mediates gene activation programs. Cell 2011;147:773-788.

-21 Tripathi V, Ellis JD, Shen Z, Song DY, Pan Q, Watt AT, Freier SM, Bennett CF, Sharma A, Bubulya PA, Blencowe BJ, Prasanth SG, Prasanth KV: The nuclear-retained noncoding RNA MALAT1 regulates alternative splicing by modulating SR splicing factor phosphorylation. Mol Cell 2010;39:925-938.

-22 Zeng R, Zhang R, Song X, Ni L, Lai Z, Liu C, Ye W: The long non-coding RNA MALAT1 activates Nrf2 signaling to protect human umbilical vein endothelial cells from hydrogen peroxide. Biochem Biophys Res Commun 2018;495:2532-2538.

23 Hardie DG, Ross FA, Hawley SA: AMPK: a nutrient and energy sensor that maintains energy homeostasis. Nat Rev Mol Cell Biol 2012;13:251-262.

-24 Hardie DG, Schaffer BE, Brunet A: AMPK: An Energy-Sensing Pathway with Multiple Inputs and Outputs. Trends Cell Biol 2016;26:190-201.

25 Zhao W, Peng F, Shu M, Liu H, Hou X, Wang X, Ye J, Zhao B, Wang K, Zhong C, Xue L, Gao M, Liu Y, Zhao S: Isogambogenic Acid Inhibits the Growth of Glioma Through Activation of the AMPK-mTOR Pathway. Cell Physiol Biochem 2017;44:1381-1395.

26 Wang S, Song P, Zou MH: AMP-activated protein kinase, stress responses and cardiovascular diseases. Clin Sci (Lond) 2012;122:555-573.

27 Jeon SM, Chandel NS, Hay N: AMPK regulates NADPH homeostasis to promote tumour cell survival during energy stress. Nature 2012;485:661-665.

-28 Zimmermann K, Baldinger J, Mayerhofer B, Atanasov AG, Dirsch VM, Heiss EH: Activated AMPK boosts the Nrf2/HO-1 signaling axis--A role for the unfolded protein response. Free Radic Biol Med 2015;88:417-426.

-29 Joo MS, Kim WD, Lee KY, Kim JH, Koo JH, Kim SG: AMPK Facilitates Nuclear Accumulation of Nrf2 by Phosphorylating at Serine 550. Mol Cell Biol 2016;36:1931-1942.

-30 Xu YY, Chen FL, Ji F, Fei HD, Xie Y, Wang SG: Activation of AMP-activated protein kinase by compound 991 protects osteoblasts from dexamethasone. Biochem Biophys Res Commun 2018;495:1014-1021.

31 Guo S, Mao L, Ji F, Wang S, Xie Y, Fei H, Wang XD: Activating AMP-activated protein kinase by an alpha1 selective activator compound 13 attenuates dexamethasone-induced osteoblast cell death. Biochem Biophys Res Commun 2016;471:545-552.

32 Hardie DG: AMP-activated/SNF1 protein kinases: conserved guardians of cellular energy. Nat Rev Mol Cell Biol 2007;8:774-785.

33 Voss M, Paterson J, Kelsall IR, Martin-Granados C, Hastie CJ, Peggie MW, Cohen PT: Ppm1E is an in cellulo AMP-activated protein kinase phosphatase. Cell Signal 2011;23:114-124.

-34 Takeuchi M, Taniguchi T, Fujisawa H: Identification and characterization of nuclear localization signals of CaMKP-N. J Biochem 2004;136:183-188. 


\section{Cellular Physiology Cell Physiol Biochem 2018:51:31-45 and Biochemistry \begin{tabular}{l|l} 
DOI: 10.1159/000495159 2018 The Author(s). Published by S. Karger AG, Basel \\
Publion
\end{tabular}

Fan et al.: LncRNA MALAT1 Protects Osteoblasts from Dexamethasone

35 Takeuchi M, Ishida A, Kameshita I, Kitani T, Okuno S, Fujisawa H: Identification and characterization of CaMKP-N, nuclear calmodulin-dependent protein kinase phosphatase. J Biochem 2001;130:833-840.

-36 Xu Y, Li G, Du B, Zhang P, Xiao L, Sirois P, Li K: Hepcidin increases intracellular Ca2+ of osteoblast hFOB1.19 through L-type Ca2+ channels. Regul Pept 2011;172:58-61.

-37 Fei HD, Yuan Q, Mao L, Chen FL, Cui ZH, Tao S, Ji F: Assessment of GSK1904529A as a promising antiosteosarcoma agent. Oncotarget 2017;8:49646-49654.

-38 Wu D, Li Y, Zhang H, Hu X: Knockdown of Lncrna PVT1 Enhances Radiosensitivity in Non-Small Cell Lung Cancer by Sponging Mir-195. Cell Physiol Biochem 2017;42:2453-2466.

-39 Tang CZ, Li KR, Yu Q, Jiang Q Yao J, Cao C: Activation of Nrf2 by Ginsenoside Rh3 protects retinal pigment epithelium cells and retinal ganglion cells from UV. Free Radic Biol Med 2018;117:238-246.

40 Han Y, Liu Y, Nie L, Gui Y, Cai Z: Inducing cell proliferation inhibition, apoptosis, and motility reduction by silencing long noncoding ribonucleic acid metastasis-associated lung adenocarcinoma transcript 1 in urothelial carcinoma of the bladder. Urology 2013;81:209 e201-207.

41 Wu X, Liu D, Gao X, Xie F, Tao D, Xiao X, Wang L, Jiang G, Zeng F: Inhibition of BRD4 Suppresses Cell Proliferation and Induces Apoptosis in Renal Cell Carcinoma. Cell Physiol Biochem 2017;41:1947-1956.

42 Zhu LQ Zhen YF, Zhang Y, Guo ZX, Dai J, Wang XD: Salinomycin activates AMP-activated protein kinasedependent autophagy in cultured osteoblastoma cells: a negative regulator against cell apoptosis. PLoS One 2013;8:e84175.

43 Lu PH, Chen MB, Ji C, Li WT, Wei MX, Wu MH: Aqueous Oldenlandia diffusa extracts inhibits colorectal cancer cells via activating AMP-activated protein kinase signalings. Oncotarget 2016;7:45889-45900.

-44 Lee M, Hwang JT, Lee HJ, Jung SN, Kang I, Chi SG, Kim SS, Ha J: AMP-activated protein kinase activity is critical for hypoxia-inducible factor-1 transcriptional activity and its target gene expression under hypoxic conditions in DU145 cells. J Biol Chem 2003;278:39653-39661.

45 Li XF, Liu XM, Huang DR, Cao HJ, Wang JY: PF-06409577 activates AMPK signaling to protect retinal pigment epithelium cells from UV radiation. Biochem Biophys Res Commun 2018;10.1016/j. bbrc.2018.05.003.

46 Wagner TC, Scott MD: Single extraction method for the spectrophotometric quantification of oxidized and reduced pyridine nucleotides in erythrocytes. Anal Biochem 1994;222:417-426.

47 Brooks MM, Neelam S, Fudala R, Gryczynski I, Cammarata PR: Lenticular mitoprotection. Part A: Monitoring mitochondrial depolarization with JC-1 and artifactual fluorescence by the glycogen synthase kinase-3beta inhibitor, SB216763. Mol Vis 2013;19:1406-1412.

-48 Tang XF, Liu HY, Wu L, Li MH, Li SP, Xu HB: Ginseng Rh2 protects endometrial cells from oxygen glucose deprivation/re-oxygenation. Oncotarget 2017;8:105703-105713.

49 She C, Zhu LQ Zhen YF, Wang XD, Dong QR: Activation of AMPK protects against hydrogen peroxideinduced osteoblast apoptosis through autophagy induction and NADPH maintenance: new implications for osteonecrosis treatment? Cell Signal 2014;26:1-8.

50 Liu W, Mao L, Ji F, Chen F, Hao Y, Liu G: Targeted activation of AMPK by GSK621 ameliorates H202-induced damages in osteoblasts. Oncotarget 2017;8:10543-10552.

51 Balteau M, Van Steenbergen A, Timmermans AD, Dessy C, Behets-Wydemans G, Tajeddine N, CastanaresZapatero D, Gilon P, Vanoverschelde JL, Horman S, Hue L, Bertrand L, Beauloye C: AMPK activation by glucagon-like peptide-1 prevents NADPH oxidase activation induced by hyperglycemia in adult cardiomyocytes. Am J Physiol Heart Circ Physiol 2014;307:H1120-1133.

52 Krajka-Kuzniak V, Paluszczak J, Baer-Dubowska W: The Nrf2-ARE signaling pathway: An update on its regulation and possible role in cancer prevention and treatment. Pharmacol Rep 2017;69:393-402.

53 Suzuki T, Yamamoto M: Molecular basis of the Keap1-Nrf2 system. Free Radic Biol Med 2015;88:93-100.

54 Zhang Q, Pi J, Woods CG, Andersen ME: A systems biology perspective on Nrf2-mediated antioxidant response. Toxicol Appl Pharmacol 2010;244:84-97.

55 den Uyl D, Bultink IE, Lems WF: Advances in glucocorticoid-induced osteoporosis. Curr Rheumatol Rep 2011;13:233-240.

56 Weinstein RS: Clinical practice. Glucocorticoid-induced bone disease. N Engl J Med 2011;365:62-70.

57 Kerachian MA, Seguin C, Harvey EJ: Glucocorticoids in osteonecrosis of the femoral head: a new understanding of the mechanisms of action. J Steroid Biochem Mol Biol 2009;114:121-128. 
Cellular Physiology Cell Physiol Biochem 2018;51:31-45 \begin{tabular}{l|l|l|l|l|l}
\hline DOI: 10.1159/000495159 2018 The Author(s). Published by S. Karger AG, Basel & and Biochemistry
\end{tabular}

Fan et al.: LncRNA MALAT1 Protects Osteoblasts from Dexamethasone

58 Li W, Kong AN: Molecular mechanisms of Nrf2-mediated antioxidant response. Mol Carcinog 2009;48:91104.

59 Itoh K, Tong KI, Yamamoto M: Molecular mechanism activating Nrf2-Keap1 pathway in regulation of adaptive response to electrophiles. Free Radic Biol Med 2004;36:1208-1213. 